\title{
ON STABILITY ANALYSIS OF BEARING-ROTOR SYSTEM
}

\author{
Meng-su Li, Xu-chu Cai \\ Donghua University, Shanghai 200051,China,Email: lims@opg.cn \\ Dan Zhang \\ University of Ontario Institute of Technology, Canada,Email: Dan.Zhang@uoit.ca
}

\begin{abstract}
The rotor system of the small power unit used in ball pen tip production machine, is discussed in detail. Meanwhile, nonlinear model of the rotor system is established and the related research method is introduced. Furthermore, the stability bifurcation of nonlinear periodic solution is obtained preliminarily. Characteristic comparisons between pseudo-periodic solution and chaotic one are put forward in the paper, which will be of benefit to further engineering applications.
\end{abstract}

\section{INTRODUCTION}

The production quality of ball pen tip is directly influenced by the rotating precision of the small power shaft system of its producing equipment. How to improve its rotating precision is therefore a key problem in the pen manufacturing industry. However, due to the fact that the rotating shaft system is unstable during production, the perturbed rotor will deviate from its normal motion orbit more and more as time goes on so that there is no favorable and satisfactory method for the stability analysis of the rotor. According to the linear theory, the amplitude of the perturbed rotor center will increase indefinitely with time, but the actual fact is that the amplitude will be held after reaching a certain limited value, and then the rotor center will more in a closed orbit (as shown in Fig. 1). Obviously, this is a kind of common nonlinear phenomenon in bearing-rotor system.

This paper is attempting to analyze the stability of the restrained rotor by nonlinear method, and provide a better theoretical foundation for the design and manufacture of multi-station machine tool with small power unit for ball pen tip production.

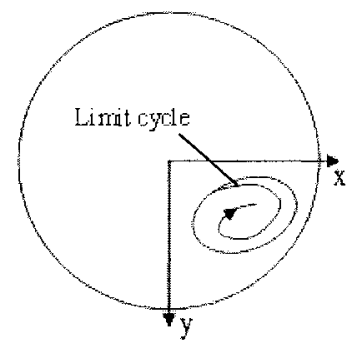

Fig. 1 Equilibrium point deviating from the limit cycle due to destabilization 


\section{NONLINEAR FORMULATION OF ROTOR SYSTEM}

Bearing-rotor system, a nonlinear dynamic system, can be described by a set of finite-dimensional, second-order and ordinary differential equations with multiparameters. Since the rotating frequency $\omega$ is the main influencing parameter, so the system that can be described only as sets of finite-dimensional, second-order and ordinary differential equations with single-parameter, is studied here. The typical form of its formulations is

$$
M f_{\mathrm{in}}(\$ q, t, \omega)=f_{\mathrm{ex}}(t, \omega) \quad(t, q) \in\left(R \times R^{m}\right)
$$

where $M$ is the mass matrix; $t$ is the time $(t \geqslant 0)$; frequency $\omega$ is a parameter on the real number axis in the system; the $m$-dimensional vector $q(t)$ is unknown; $f_{\text {in }}(\& q, t, \omega)$ is the internal force vector which results from rigidity of the uncoupled axis, force of the bearing film, etc. in the system; $f_{\text {ex }}(t, \omega)$ represents the external excitation force vector acting on the system.

Introducing the state variable, $u=\left(\begin{array}{c}q \\ q\end{array}\right)$, then we can obtain the expression of the system equation in state space,

$$
\frac{\mathrm{d} u}{\mathrm{~d} t}=F(t, \omega, u), \quad(t, u) \in\left(R \times R^{m}\right),
$$

where

$$
F(t, \omega, u)=\left[\begin{array}{c}
\& \\
M^{-1}\left\{-f_{\mathrm{in}}(\& q, t, \omega)+f_{\mathrm{ex}}(t, \omega)\right\}
\end{array}\right] .
$$

If there is no time variable $t$ in $F$, and satisfying $F(t, \omega, u) \stackrel{\text { def }}{=} F(\omega, u) \neq 0$, then Eq. (2) can be simplified as follows,

$$
\frac{\mathrm{d} u}{\mathrm{~d} t}=F(\omega, u), \quad(t, u) \in\left(R \times R^{2 m}\right)
$$

Now, the nonlinear bearing-rotor dynamic system, corresponding to Eq. (3), will then be autonomous.

If $F$ is a periodic function of time $t$, namely satisfying $F(t, \omega, u)=F(t+T, \omega, u)$, and $F(t, \omega, 0) \neq 0$, then Eq. (2) can be simplified as follows,

$$
\frac{\mathrm{d} u}{\mathrm{~d} t}=F(t, \omega, u)=F(t+T, \omega, u), \quad(t, u) \in\left(R \times R^{2 m}\right)
$$

The nonlinear bearing-rotor dynamic system is then nonautonomous.

\section{STABILITY ANALYSIS OF THE NONLINEAR SYSTEM}

\subsection{Mapping}

The physical model of bearing-rotor system can be expressed in the forms of Eqs. (1), (2). It is therefore of great significance to study the problems of stability bifurcations of steady-state solutions for this type of system. 
If the problems, such as the stability, bifurcations and regions of attraction of the steady-state periodic solutions, are studied directly through the nonlinear dynamic system Eq. (2), it will be quite difficult to obtain the analytic expression of the periodic solution $u(t)$ in phase space, while the using of closed orbit comprised of infinite number of points will also bring comparative difficulties to numerical analysis. Therefore, it is necessary to find a much better way to express $u(t)$. As the nonlinear mapping expression, based on Eq. (1) of the nonlinear dynamic system and its manifold $\varphi_{t}$, can be given as follows,

$$
u^{(n+1)}=G\left(u^{(n)}\right) \text { or } u \rightarrow G(u)
$$

where $n=2 m, G=\varphi$ is a vector field, and we here are concerned only about the characteristics of the steady-state periodic solutions $u(t)=u(t+T)$, so a point mapping system, converted from the nonlinear dynamic system, will thus be put forward for more convenient studied as follows.

For the autonomous system Eq. (3) deduced from the Eq. (2), i. e.,

$$
\varphi_{t}: \quad \frac{\mathrm{d} u}{\mathrm{~d} t}=F(\omega, u), \quad(t, u) \in\left(R \times R^{n}\right),
$$

assume that $u(t)$ is a periodic orbit of the manifold field $\varphi_{t}$ defined in $R^{n}$, namely $u(t)$ is a periodic solution of the above equation. Taking a global cross sectional hypersurface $\sum \subset R^{n}$ with $n$-1 dimensions in the $n$-dimensional state space, this hypersurface $\sum$ doesn't have to be a hyperplane but it should intersect the manifold field $\varphi_{t}$ everywhere.

Thus, the number of intersections, formed by the periodic solution $u(t)$ and $\Sigma$ from the same direction ( $u(t)$ goes across $\sum$ on its same side and forms the intersections) is finite. If the number of intersections is $K$, then $u(t)$ is the KT periodic solution of the system.

Therefore, the mapping $P$ of point $q \subseteq \sum$ can be defined as:

$$
P(q)=\varphi_{\tau}(q)
$$

where $\tau=\tau(q)$ is the time used by manifolds $\varphi_{t_{0}}(q)$ when first return to $\Sigma$ in the same direction with $q$ as the starting point. Note that some other manifolds $\varphi_{t_{0}}(A)$, with certain point $A$ on $\sum$ as their corresponding starting point may never return to $\sum$ in the same direction, which represents the corresponding orbits of the divergent transient state. These points and their corresponding orbits in transient state will be disregarded and defined only as puzzled points on $\sum$ without further study anymore.

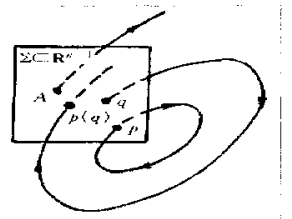

Fig. 2 Mapping of an autonomous system 
For the autonomous system Eq. (4) as deduced from Eq. (2),

$$
\varphi_{t}: \quad \frac{\mathrm{d} u}{\mathrm{~d} t}=F(t, \omega, u)=F(t+T, \omega, u) \quad(t, u) \in\left(R \times R^{n}\right),
$$

if now taking the time $t$ as a state variable, the above equation can then be converted into the form of autonomous system Eq. (3) by adding one more dimension,

$$
\left\{\begin{array}{l}
\frac{\mathrm{d} u}{\mathrm{~d} t}=F(\theta, \omega, u) \\
\frac{\mathrm{d} \theta}{\mathrm{d} t}=1 \quad(t, \theta, u) \in\left(R \times S^{1} \times R^{n}\right)
\end{array}\right.
$$

$S^{1} \times R^{n}$ is the form of manifolds in phase space, and the circle component $S=R(\bmod T)$ shows the periodic dependence of vector field $F$ on the variable $\theta$. Then a global cross section can be defined as follows,

$$
\Sigma=\left\{(\theta, u) \in S^{1} \times R^{n} \mid \theta_{0} \theta_{0}\right\}
$$

Now, all solutions $(\theta, u)$ will intersect with $\Sigma$. The mapping $P: \Sigma \rightarrow \Sigma$ of point $q \in \sum$ can therefore be defined as:

$$
P(q)=u\left(\theta_{0}+T, q\right)
$$

where $u(t, q)$ is a solution of a nonautonomous system, (as shown in Fig. 3) with $u\left(\theta_{0}, q\right)=u_{0}$ as its starting point. Note that it differs from the autonomous system, and there will be no more puzzled point $A$ on the cross sectional surface $\Sigma$ now.

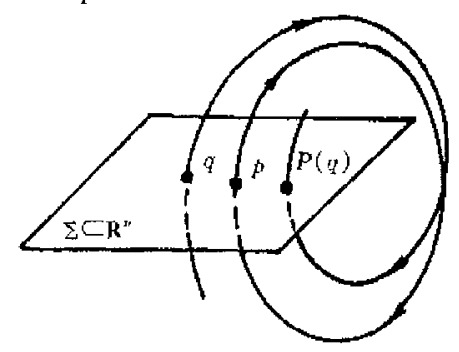

Fig. 3 Mapping of a nonautonomous system

Although the methods adopted to set up a point-mapping system, are somehow different between autonomous system Eq. (3) and nonautonomous system Eq. (4), yet a completely same point-mapping system in form can be obtained finally as follows, without any distinction

$$
u^{(k+1)}=P\left(u^{(k)}\right) \quad k \in Z \text { or } u \rightarrow P(u)
$$

It is obvious that the intersections $p_{1}, p_{2}, \Lambda, p_{k}$, formed by $\mathrm{KT}$ periodic solutions $u(t)=u(t+k T), k \in Z$ with the cross section supersurface $\sum$, are all the P-K periodic points of mapping $P$, according to the definition mentioned above.

$$
P^{k}\left(p_{i}\right)=p_{i}, \quad i=1,2, \Lambda, k
$$


The domain of attraction of these P-K periodic points $p_{i}(i=1,2, \Lambda, k)$ on the supersurface $\Sigma$, is just that part of the attraction domain of the solutions $u(t)=u(t+k T)$ in the state space intersected by the supersurface $\Sigma$, whose stability with respect to the mappings $P$ reflects the stability of the solutions $u(t)=u(t+k T)$ with respect to the manifold $\varphi_{t}$.

Only the points on the supersurface $\sum$ and their mapping rules are concerned in the mapping system established on the dynamic system, while the characteristics of all other points on the solution orbits in the entire global state space are not concerned. The study of the problem concerned is thus much simplified, which is advantageous for the further application of numerical methods.

\subsection{Stability analysis}

It can be seen from the above discussion that a discrete dynamic system can be transformed from the continuous dynamic system by means of mapping.

$$
u^{(k+1)}=P\left(u^{(k)}\right) \quad k \in Z \text { or } u \rightarrow P(u)
$$

Consequently, the analysis of continuous dynamic system of Eq. (2) can be obtained through analyzing the discrete dynamic system of Eq. (12).

Assuming there is a stable solution $u^{t}(t)$ of the dynamic system Eq. (3), then $u^{e}(t)$ will be unstable if the initial perturbations $u^{e}(t)$ increase with time; or $u^{e}(t)$ is stable during perturbations if it is gradually damped. The stability theory related to the infinitely small perturbations is actually a linear theory, since the high-order terms can be ignored in comparison with the linear terms in a perturbation equation. Assuming that $u^{e}(t, \omega)$ is a stable solution of dynamic system Eq. (2), and $\delta v$ is a randomly given constant perturbation of $u$, then we shall obtain:

$$
\delta \frac{\mathrm{d} v}{\mathrm{~d} t}=F(t, \omega, u+\delta v)-F(t, \omega, u)
$$

When $\delta \rightarrow 0$, the perturbation tends to be infinitely small, then we can get

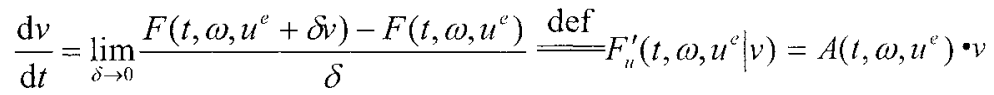

where $A\left(t, \omega, u^{e}\right)=F_{u}^{\prime}\left(t, \omega, u^{e} \mid \bullet\right)$, is a linear operator of the variable $v$ after the vertical line. It is an $n \times n$ matrix function for the $n$-dimensional problems. For nonlinear autonomous system Eq. (3), Eq. (14) can be simplified as,

$$
\frac{\mathrm{d} v}{\mathrm{~d} t}=F_{u}^{\prime}\left(\omega, u^{e} \mid v\right)=A\left(\omega, u^{e}\right) \cdot v
$$

For the fixed point $u^{*}$ of the discrete dynamic system in Eq. (12), we obtain,

$$
u^{*}+\delta v_{k+1}=P\left(u^{(k)}+\delta v_{k}\right)
$$

where

$$
\begin{gathered}
u^{*}=u^{e}\left(t_{k}, \omega\right)=u^{e}\left(t_{k}+T, \omega\right) \\
v_{k}=v\left(t_{k}\right) ; v_{k+1}=v\left(t_{k}+T\right)
\end{gathered}
$$

We can therefore obtain the following results with respect to the perturbation when $\delta \rightarrow 0$ :

$$
u^{*}+\delta v_{k+1}=P\left(u^{*}\right)+P_{1 t}^{\prime} \cdot \delta v_{k}
$$


so

$$
v_{k+1}=P_{t \prime}^{\prime} \cdot v_{k} \quad k \in Z
$$

$P_{u}^{\prime}$, the linear form of the fixed point $u^{*}$ of the mapping, is a constant matrix here.

It can be proven that Eqs. (14), (17), completely equivalent to each other, are perturbation equations in continuous and discrete forms respectively. The stability of the stable solution $u^{e}(t, \omega)$ in the system can be evaluated through discussing the perturbation equations.

For the autonomous system Eq. (3), when the outer parameter $\omega$ is a certain value of $\omega_{m}$, these following condition can be satisfied,

$$
F\left(\omega_{m}, u^{s}\right)=0 \quad u^{s} \in R^{n}
$$

where $u^{s}$ is the equilibrium point of the system, so it is considered that the stability of the equilibrium point solutions can be judged by the eigenvalues of the matrix $A\left(\omega_{m}, u^{s}\right)=F_{u}^{\prime}\left(\omega_{m}, u^{s} \cdot\right)$ which is obtained when the linear system is at $u^{s}$. Here the matrix is an $n \times n$ constant one.

$$
\frac{\mathrm{d} v}{\mathrm{~d} t}=F_{u}^{\prime}\left(\omega, u^{s} \mid v\right)=A\left(\omega, u^{s}\right) \cdot v
$$

The loss of stability of system Eq. (3), as a result from the destabilization of the equilibrium point with the changing of outer parameter $\omega$, will cause bifurcation phenomenon with multifarious forms. Since destabilization of the equilibrium point solutions is one of the main forms of bifurcation in bearing-rotor system, so we study only this form of bifurcation of equilibrium points here. The distinguishing criteria are as follows.

Assuming $\omega=\omega_{c}$, the equilibrium point solution $u^{s}$ of the autonomous system Eq. (3) satisfies that:

(1) $F(\omega, u)$ is differentiable in the domains of $\left(\omega_{c}, u^{*}\left(\omega_{c}\right)\right)$;

(2) There is a pair of pure imaginary nonzero eigenvalues $\pm \mathrm{i} \beta_{0}\left(\beta_{0}>0\right)$ in the matrix $F_{i l}^{\prime}\left(\omega_{c}, u^{s}\left(\omega_{c}\right) \mid \cdot\right)$ obtained when $F(\omega, u)$ is at the stable solution $u^{\prime \prime}\left(\omega_{c}\right)$, while the other $n-2$ eigenvalues all have negative real parts.

(3) $\left.\frac{\mathrm{d} \alpha}{\mathrm{d} \omega}\right|_{\omega=\omega_{c}} \neq 0$, where $\alpha(\omega) \pm \mathrm{i} \beta(\omega)$ is the continuous unfolding eigenvalues obtained by $\left.F_{n}^{\prime}\right|_{\omega}=\omega c$ with respect to $\pm \mathrm{i} \beta_{0}$. In this case, the equilibrium point solutions are destabilized. A non-constant periodic solution $u^{H}$, corresponding to the self-exited limit cycle of the system, will be bifurcated from steady-state equilibrium point solutions of $u^{s}\left(\omega_{c}\right)$ at $(\omega, u(\omega))=\left(\omega_{c}, u^{s}\left(\omega_{c}\right)\right)$ in the autonomous system.

According to the different periodic solutions due to bifurcation, it can be further classified into two different cases: supercritical condition and bifurcation, to be described as follows, 
(1) If $\omega>\omega_{c}$, a stable periodic solution $u^{H}$ is bifurcated from the equilibrium point solution $u^{s}\left(\omega_{c}\right)$ of the system. The periodic solution $u^{I \prime} \rightarrow u^{s}\left(\omega_{c}\right)$ is a supercritical bifurcation obtained when $u^{\prime \prime} \rightarrow u^{s}\left(\omega_{c}\right)$. Its bifurcation is characterized by the gradually varying periodic solution with the changing of $\omega$, and there is no phenomenon of "jump and decay" in this system, as shown in Fig. $4(\mathrm{a})$.

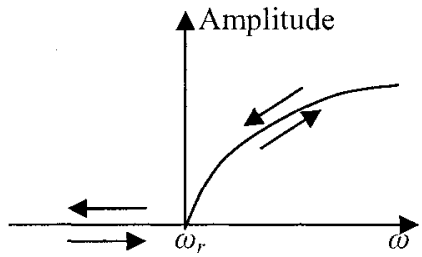

(a) Supercritical bifurcation

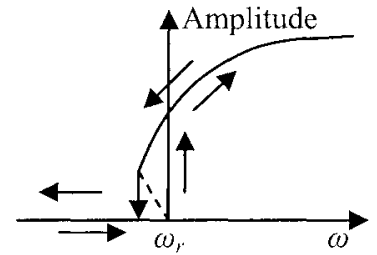

(b) Subcritical bifurcation

Fig. 4 Bifurcations on equilibrium point solutions

(2) If $\omega<\omega_{c}$, an unstable periodic solution $u^{H}$ (with $u^{H} \rightarrow u^{s}\left(\omega_{c}\right)$ when $\left.\omega \rightarrow \omega_{c}\right)$ is bifurcated from the equilibrium solution $u^{s}\left(\omega_{c}\right)$ in the system. Its bifurcation is characterized by the abrupt change of periodic solution with the changing of $\omega$, with the phenomenon of "jump and decay" because there often exists a stable periodic solution besides the unstable periodic ones, as shown in Fig. $4(b)$.

The matrices in the corresponding perturbation Eq. (14) are constant ones, according to the above results. Therefore the perturbation of the stable equilibrium point solutions in the autonomous system Eq. (3) can be directly confirmed by analyzing the Eq. (14).

\subsection{Stable perturbation of the nonlinear steady-state periodic solutions}

For a given outer parameter $\omega$ and its corresponding steady-state periodic solution $u_{p}$ with period $T$, (i. e., $u_{p}=u_{p}(t+T)$ ), its perturbation equation is as follows,

$$
\frac{\mathrm{d} v}{\mathrm{~d} t}=F_{u}^{\prime}\left(t, \omega, u_{p} \mid v\right)=A(t) \cdot v,
$$

where $A(t)=A(t+T)$ is a matrix function with period $T$. The perturbation equation related both to steady-state periodic solutions in an autonomous system and to a periodic nonautonomous one can be represented by Eq. (20)

If $V(t)$ is a fundamental solution matrix of Eq. (20), there must exist a nonsingular matrix $\Phi(t)=\Phi(t+T)$ with period $T$ and a constant matrix $D$, so that

$$
V(t)=\Phi(t) \cdot e^{t D}
$$

Meanwhile, according to the periodic property of $A(t)$ in Eq. (20), we should have

$$
\frac{\mathrm{d} V(t+T)}{\mathrm{d} t}=A(t+T) \cdot V(t+T)=A(t) \cdot V(t+T)
$$


consequently, $V(t+T)$ is also the fundamental solution matrix of Eq. (20). From Eq. (20) we can have

$$
V(t+T)=\Phi(t+T) \cdot e^{(t+D) D}=\Phi(t) \cdot e^{t D} \cdot e^{T D}=V(t) \cdot e^{T D}
$$

The above equation can also be simplified as

$$
V(t+T)=V(t) \cdot C
$$

where $C=e^{T D}$ is a constant matrix.

Take any two fundamental solution matrices $V_{1}(t)$ and $V_{2}(t)$ in Eq. (20), then the constant matrices $C_{1}$ and $C_{2}$ can be obtained correspondingly, so that

$$
\left\{\begin{array}{l}
V_{1}(t+T)=V_{1}(t) \cdot C_{1} \\
V_{2}(t+T)=V_{2}(t) \cdot C_{2}
\end{array}\right.
$$

Meanwhile, there must exist nonsingular constant matrix $\mathrm{S}$, which satisfies:

$$
V_{2}(t)=V_{1}(t) \cdot S
$$

Therefore

$$
V_{2}(t+T)=V_{1}(t+T) \cdot S=V_{1}(t) \cdot C_{1} S=V_{2}(t) \cdot S^{-1} C_{1} S
$$

That is:

$$
C_{2}=S^{-1} C_{1} S
$$

Consequently, $C$ as well as $D$, is a group of similar constant matrices. Namely their eigenvalues are independent of the choice of given initial conditions and fundamental solution matrices, but are determined only by $A(t)$ in Eq. (20). $C$ and $D$, for discrete and for continuous conditions respectively, are the transfer matrices of Eq. (20). Their eigenvalues $\lambda$ and $\delta$ are respectively the multiplier and the exponent index. From Eqs. (21), (22) we can see that they will determine the stability of Eq. (20) with respect to the origin.

From the relations between $C$ and $D$, we can obtain

$$
\lambda=e^{\sigma T}=e^{\operatorname{Re}(\sigma T)} \cdot[\cos (\operatorname{Im}(\sigma T))+\mathrm{i} \cdot \sin (\operatorname{Im}(\sigma T))]
$$

and

$$
\left\{\begin{array}{l}
\operatorname{Re}(\sigma)=\frac{1}{T} \cdot \ln |\lambda| \\
\operatorname{Im}(\sigma)=\frac{1}{T} \cdot \arctan \left[\frac{\operatorname{Im}(\lambda)}{\operatorname{Re}(\lambda)}\right]
\end{array}\right.
$$

Note that $\sigma$ has multiple values, and should be taken in the region $0 \leqslant \operatorname{Im}(\sigma) \leqslant \frac{2 \pi}{T}$ for practical applications.

Furthermore, the stability of any solutions of Eq. (20) with respect to the origin can be judged by $\lambda$ and $\sigma$. The stability criteria and bifurcation conditions of the steady-state periodic solutions can also be obtained then in the dynamic system of Eq. (2).

Assume that the basic solution matrix $V(t)$ is a unit matrix at the initial time $t_{0}$, that is

$$
V\left(t_{0}\right)=I
$$


so $\quad V\left(t_{0}+T\right)=V\left(t_{0}\right) \cdot C=C$

Assume that $v(t)$ is a perturbation at the time $t$, and $v(t+T)$ is another perturbation after a period of $T$, then:

$$
\begin{gathered}
v\left(t_{0}\right)=V\left(t_{0}\right) \cdot \varphi_{i}=\varphi_{i} \quad \varphi_{i} \in R^{n} \\
v\left(t_{0}+T\right)=V\left(t_{0}+T\right) \cdot \varphi_{i}=V\left(t_{0}\right) \cdot C \cdot \varphi_{i}=C \cdot \varphi_{i} \quad \varphi_{i} \in R^{n}
\end{gathered}
$$

so

$$
S_{i}=\frac{\left\|v\left(t_{0}+T\right)\right\|}{\left\|v\left(t_{0}\right)\right\|} \leqslant\|C\|
$$

Assume $S_{\max }$ is the maximum of $S_{i}$, and thus

$$
S_{\max }=\|C\|=\max _{i=1, \wedge, n}\left(\left|\lambda_{i}\right|\right)
$$

Then we can get the following conclusions:

(1) The sufficient condition for the stability of the periodic solution $u_{p}$ in the dynamic system of Eq. (2), is that its maximum multiplier modulus is less than 1, namely all multipliers are within the unit circle on complex plane.

(2) $u_{p}$ is unstable when the maximum multiplier modulus is greater than 1 .

(3) $u_{p}$ is critical steady state when the maximum multiplier modulus is equal to 1 .

Meanwhile, similar to the equilibrium point solution, there may be destabilization with multifarious bifurcation in the periodic solutions of the dynamic system of Eq. (2). Further study shows that the forms of the destabilization and bifurcation of the periodic solution $u_{p}$, according to different positions where the maximum multipliers of the modulus pass through the unit circle, can be classified into three kinds as follows:

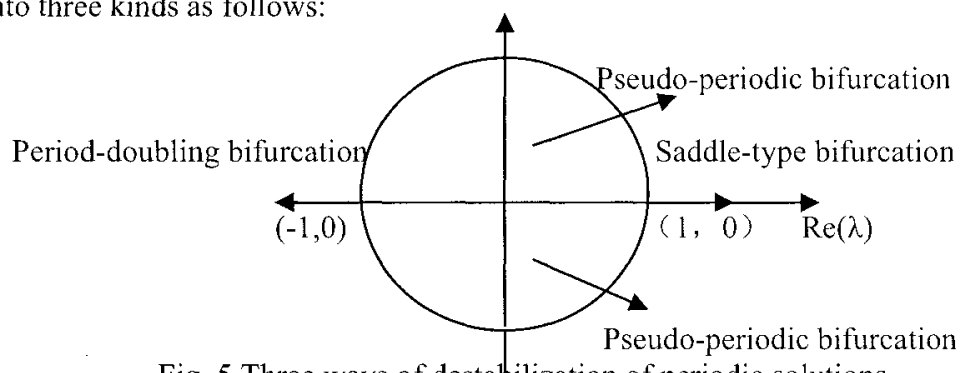

Fig. 5 Three ways of destalilization of periodic solutions with indications of multipliers

(1) When the maximum multiplier of modulus passes through the unit circle on the point of $(1,0)$, the possible ways of destabilization and bifurcation of periodic solutions may involve saddle-type, fork-type, symmetrical breakage- type and so on, depending on different systems.

(2) When the maximum multiplier of the modulus passes through the unit circle on the point of $(1,0)$, the periodic solution will be destabilized via a perioddoubling bifurcation. After passing through the bifurcated point, its period will split 
from $T$ into $2 T$, and then $4 T$ on the orbits. This kind of period-doubling bifurcation will result in the chaotic motion in the system finally.

(3) When the maximum multipliers of a pair of moduli pass through the unit circle in a form of conjugate complex numbers (the imaginary part is nonzero), the pseudo-periodic solutions will be periodically bifurcated and obtained on the basis of period solutions.

\subsection{Nonlinear pseudo-periodic and chaotic solutions}

Besides the equilibrium points and periodic solutions, the pseudo-periodic and chaotic solutions are two other kinds of steady-state motion forms in the nonlinear dynamic system of Eq. (2), and are comparatively more complex. It should be pointed out that there is a direct relation between the periodic solution and these two kinds of steady-state motion in pseudo-periodic and chaotic solutions. Pseudoperiodic solution in general is periodically bifurcated from the basis of periodic solution, and so also are the three ways of occurrences of chaotic solutions. The period-doubling bifurcation is actually a processing of continuous solution-cracking and period-doubling, and finally becoming chaotic. On the other hand, the pseudoperiodic bifurcated ways can result from such course as: firstly being bifurcated to pseudo-periodic solutions and then generating chaotic ways on the basis of period solutions, while the intermittent form behaves as the alternate appearances of periodic and chaotic solutions. Table 1 is the comparison between the characteristics of periodic, pseudo-periodic and chaotic solutions.

\section{CONCLUSION}

The bifurcation and regularities of stability variation with the changing of rotating speed $\omega$, are obtained based on the detailed partial analysis on the stability problems of both stable and unstable bearing-rotor system. There will be more than a single periodic solution with the same rotating speed $\omega$ in practice. Which solution the system is carrying out will depend on initial conditions. Therefore, it is necessary to study the influence on system solutions due to initial conditions, namely by finding the domains of attraction of every solution in the system. In addition, the study shows that no matter whether the nonlinear bearing-rotor system is, a stable one or not, there are definite relations between destabilized attenuation and the orbits of periodic motion of the system, whenever there exists a certain orbit of the rotor center. Different attenuation rates can be obtained when the system is destabilized at different positions.

\section{REFERENCES}

1. Wen Xi-sen, et al, Modeling and Dynamic Analysis of Mechanical System, Science Press, Beijing, China, 11, 2004.

2. Iooss G.., Joseph D. D., Elementary Stability and Bifurcation Theory, Springer-Verlag, New York, 1980.

3. Lund J. W., Saibel E., Oil-whip Whirl Orbits of a Rotor in Sleeve Bearings. Journal of Engineer Industry, ASME, 89, 1967.

4. Gunter E. J., Hamphris R. R., Springeer H., Influence of Unbalanced on the Nonlinear Dynamical Response and Stability Flexible Rotor Bearing System. Rotor Dynamical Instability, ASME, 1983.

5. Liu Heng, Chen Shao-ding, PMC Method in the Global Nonlinear Analysis and Its Application in Bearing-rotor System. Journal of Applied Mechanics (in Chinese), 12(5), 1995. 